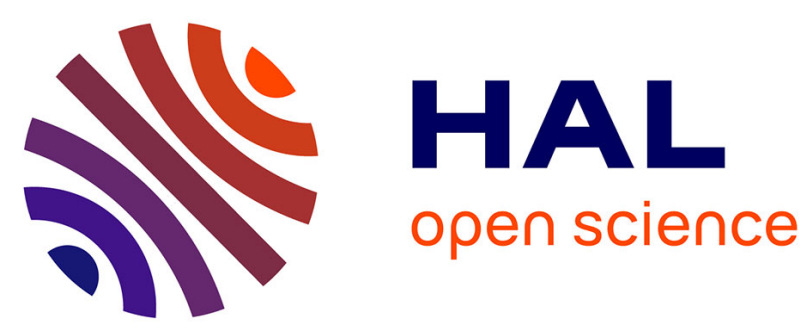

\title{
Compliance with recommendations and clinical outcomes for formal and informal infectious disease specialist consultations
}

Elodie Sellier, José Labarère, Stéphane Gennai, G. Bal, Patrice François, P. Pavese

\section{To cite this version:}

Elodie Sellier, José Labarère, Stéphane Gennai, G. Bal, Patrice François, et al.. Compliance with recommendations and clinical outcomes for formal and informal infectious disease specialist consultations. European Journal of Clinical Microbiology and Infectious Diseases, 2011, 30 (7), pp.887-894. 10.1007/s10096-011-1172-7 . hal-00669039

\section{HAL Id: hal-00669039 \\ https://hal.science/hal-00669039}

Submitted on 11 Feb 2012

HAL is a multi-disciplinary open access archive for the deposit and dissemination of scientific research documents, whether they are published or not. The documents may come from teaching and research institutions in France or abroad, or from public or private research centers.
L'archive ouverte pluridisciplinaire $\mathbf{H A L}$, est destinée au dépôt et à la diffusion de documents scientifiques de niveau recherche, publiés ou non, émanant des établissements d'enseignement et de recherche français ou étrangers, des laboratoires publics ou privés. 


\section{Original Article}

Title: Compliance with Recommendations and Clinical Outcomes for Formal and Informal Infectious Disease Specialist Consultations.

Elodie Sellier ${ }^{1,2}$, José Labarère ${ }^{1,2}$, Stéphane Gennai ${ }^{3}$, Gaëlle Bal ${ }^{1}$, Patrice François ${ }^{1,2}$, Patricia Pavese ${ }^{3}$

1. Quality of Care Unit, University Hospital, F-38043, Grenoble, France

2. Techniques de l'Ingénierie Médicale et de la Complexité (TIMC), Unité Mixte de Recherche 5525, Centre National de la Recherche Scientifique (CNRS), Université Joseph Fourier - Grenoble 1, F-38041, Grenoble, France.

3. Department of Infectious Diseases, University Hospital, F-38043, Grenoble, France

Corresponding author: $\quad$ Elodie Sellier

Quality of Care Unit

Pavillon Taillefer

CHU BP 217

38043 Grenoble Cedex 9

France

telephone: 33476765346 / fax: 33476768831

ESellier@chu-grenoble.fr / elodie.sellier@gmail.com 


\section{ABSTRACT}

Purpose: To compare compliance with recommendations and clinical outcomes between formal and informal infectious disease specialist consultations.

Methods: 627 consecutive adult inpatients who received an infectious disease consultation in a university-affiliated hospital were included. After adjusting for quintile of propensity score, we compared compliance with the consultant's recommendations and clinical outcomes for $443(70.7 \%)$ and $184(29.3 \%)$ formal and informal consultations.

Results: Informal and formal consultations were associated with comparable levels of compliance with recommendations for antimicrobial treatment $(86.5 \% v 88.9 \%$; adjusted odds ratio $[\mathrm{aOR}], 0.63 ; 95 \%$ confidence interval, $0.34-1.14 ; P=0.13)$ and diagnostic or monitoring tests $(72.6 \% \vee 72.0 \%$; aOR, $0.91[0.53-1.57] ; P=0.73)$. The rates of early clinical improvement (58.2\% v 58.6\%; aOR, 1.11 [0.70-1.74]; $P=0.66)$, subsequent consultation (34.2\% v 36.3\%; aOR, 0.80 [0.53-1.21]; $P=0.29)$, in-hospital mortality (4.9\% v 8.4\%; aOR, 0.55 [0.24-1.24]; $P=0.15$ ), and the median length of stay (23 $v 20$ days; aOR of discharge, $0.90[0.74-1.10] ; P=0.30)$ did not differ depending on the type of consultation.

Conclusions: This study provides observational evidence that informal consultations result in levels of compliance with recommendations comparable to formal consultations, without compromising patient safety. Further study is needed to refine the criteria for requesting or providing informal rather than formal consultations.

Key words: Consultations; Specialists; Adherence; Safety; Inpatients 


\section{INTRODUCTION}

A substantial part of infectious disease specialists' workload stems from informal consultations, which refers to the process whereby a clinician obtains advice on a patient or clinical question from a colleague with a particular clinical expertise [1-3]. In contrast with formal consultation, the consultant does not directly examine the patient and therefore his recommendations are based exclusively on information provided by the requesting physician $[4,5]$.

Informal consultation has many advantages and is often considered a convenient alternative to formal consultation by both requesting and consulting physicians $[1,6]$. The most common reasons cited by physicians for requesting informal consultation include direct contact with the consultant, saving time in obtaining information, saving money for the patient or a third-party payer, and remaining up to date with current medical knowledge $[4,7]$. From the perspective of consulting physicians, informal consultation provides an opportunity to educate colleagues, identify topics for continuing medical education and guideline development, and facilitate future formal consultation $[1,7,8]$. Yet informal consultation has certain drawbacks, including the lack of compensation for the consultant, increased risk of legal liability, and inaccuracy and incompleteness of the information exchanged $[1,6,8,9]$.

Previous studies have documented the time spent, type, and content of informal consultations in infectious disease practice [10-13]. Surprisingly, little is known about the effectiveness and safety of informal consultations. One measure of the effectiveness of a consultation is compliance with the consultant's recommendations, whereas patient clinical outcomes are likely to reflect the efficacy and safety of a consultation $[15,16]$. The present 
study aimed to compare compliance with recommendations and clinical outcomes between formal and informal infectious disease specialist consultations. 


\section{METHODS}

\section{Study design.}

We conducted a post-hoc analysis of a prospective cohort study that evaluated the outcomes associated with the type (i.e., formal versus informal) of solicited infectious disease consultations for adult inpatients. The rationale, design, and primary outcomes of the original study have been detailed elsewhere [17]. The institutional review board approved the protocol and waived the requirement for informed consent. The present manuscript complied with the Strengthening the Reporting of Observational Studies in Epidemiology (STROBE) checklist [18].

\section{Patients.}

Infectious disease specialists at a 2,200 bed university-affiliated hospital recruited consecutive patients between December 5, 2007 and June 5, 2008. Eligible patients were inpatients 18 years of age or older for whom an infectious disease specialist consultation was requested. Patient exclusion criteria were previous enrollment in the study and discharge within $24 \mathrm{~h}$ of consultation. Patients were followed up until death or discharge, whichever occurred first.

\section{Data collection.}

Using a standardized case report form, the consulting infectious disease specialist prospectively documented the type, reason, and recommendations for the index and 
subsequent consultations. A physician collected information on demographics, baseline comorbid diseases, vital signs, and laboratory findings from a structured chart review. For each patient, we retrospectively computed the Charlson comorbidity index score based on the standardized definitions and weights for the 19 comorbid diseases that comprise this score [19].

\section{Formal versus informal consultation.}

The infectious disease consultation service was available $24 \mathrm{~h}$ a day, 7 days a week. A board-certified infectious disease specialist and a resident provided coverage during working hours and five board-certified specialists provided overnight and weekend coverage.

The route of consultation was categorized as formal and informal. Formal consultations were defined as bedside consultations with clinical examination of the patient by the infectious disease specialist and recommendations reported in the medical chart. Conversely, informal consultations consisted of face-to-face, telephone, or e-mail interactions between the treating physician and the infectious disease specialist, without the patient being examined by the infectious disease specialist. Face-to-face and telephone consultations resulted in oral recommendations, whereas e-mail consultations involved recommendations that were provided by e-mail. Requesting infectious disease specialist consultation was left to the discretion of the physician and there was no policy recommendation regarding the route of consultation in this study.

\section{Study outcomes.}


The primary effectiveness outcome was compliance with the recommendations of the infectious disease specialist [16]. A physician, independent of those in charge of the patients, searched for evidence of compliance with the recommendations for treatment and diagnostic or monitoring tests. Recommendations had to have been implemented within $48 \mathrm{~h}$, unless the infectious disease specialist specified a different time frame. Compliance with recommendations for treatment was ascertained based on antibiotic agents, dosage, timing, and route of administration. Compliance with recommendations for performing diagnostic or monitoring tests was ascertained based on imaging or laboratory tests ordered or performed. In accordance with previous studies [16], consultations resulting in no recommendation were omitted from the compliance analysis.

Patient clinical outcomes included early clinical improvement, subsequent consultations, length of stay, and in-hospital mortality. Early clinical improvement was defined as symptomatic recovery, resolution of abnormalities in vital signs, return to normal mental status, and normalization of the white blood cell count $72 \mathrm{~h}$ after the index consultation. This time frame was determined based on previous studies [20, 21]. Resolution of abnormalities in vital signs was ascertained using the following cut-offs for the highest temperature $\left(\leq 37.8^{\circ} \mathrm{C}\right)$, heart rate $(\leq 100$ beats $/ \mathrm{min})$, and respiratory rate $(\leq 24$ breaths $/ \mathrm{min})$, and the lowest systolic blood pressure $(\geq 90 \mathrm{mmHg})$ and oxygen saturation $(\geq 90 \%)$. Patients who were asymptomatic at baseline and those who were recommended antibiotic prophylaxis were omitted from the analysis of early clinical improvement.

Information on compliance with recommendations and patient clinical outcomes were obtained from a structured chart review and computerized hospital databases. We attempted to limit the potential for assessment bias by using standardized criteria. Moreover, outcomes were ascertained by a physician who was unaware of the hypotheses of this analysis. 


\section{Statistical analyses.}

Baseline characteristics were reported as percentages for categorical variables and median and interquartile range (i.e., $25^{\text {th }}$ and $75^{\text {th }}$ percentiles) for continuous variables. To compare patient characteristics and consultation patterns between formal and informal consultations, we performed Wilcoxon rank sum tests for continuous variables and chi-square tests or Fisher exact tests when appropriate for categorical variables.

We used univariable logistic regression to estimate the unadjusted odds ratios of binary outcomes associated with informal consultation. A discrete time logistic hazard model was used to examine the association between the length of stay and the type of consultation. The length of stay was censored at the time of death for patients who died in the hospital. Lower odds of discharge corresponded to a longer length of stay.

Because the type of consultation was not randomly assigned in this observational study, unadjusted comparisons of outcomes for patients who received formal and informal consultations might be confounded by imbalances in baseline characteristics. Consequently, we performed propensity score analysis that compensated for differences in measured patient baseline characteristics between formal and informal consultations [22, 23]. Conceptually, our propensity score corresponded to the conditional probability of exposure to an informal consultation given observed baseline characteristics. Stratifying patients who received formal and informal consultations on the propensity score tended to balance all observed characteristics that were used to construct the score, thus approximating the conditions of random treatment assignment $[22,23]$. We derived the propensity score using a full nonparsimonious logistic regression model that included all baseline characteristics and comorbid diseases listed in Tables 1 and 2 as covariates. This model yielded a c-statistic of 0.80 , indicating a satisfactory ability to differentiate patients who received formal and 
informal consultations. Each patient was assigned a propensity score, which ranged from 0.05 to 0.99 and reflected the conditional probability of receiving an informal consultation given his baseline characteristics. Patients were stratified by quintiles of propensity score [22, 23]. We then estimated the odds ratios of binary outcomes associated with informal consultation after adjusting for the quintile of propensity score.

Two-sided $P$-values less than 0.05 defined statistical significance. All analyses were performed with Stata 10.0 (Stata Corp, College Station, TX, USA). 


\section{RESULTS}

A total of 1049 solicited infectious disease specialist consultations were recorded during the 6-month enrollment period (Figure). Of these, 388 consultations involved patients who were already enrolled in the study and one consultation involved a patient who was discharged within $24 \mathrm{~h}$ of enrollment. Thirty-three additional patients were excluded from the study because of missing chart information. Finally, our analytical sample consisted of 627 patients, including $443(70.7 \%)$ and 184 (29.3\%) formal and informal consultation recipients, respectively. Informal consultations were requested by phone (154 [83.7\%]), in face-to-face interactions (27 [14.7\%]), and by e-mail (three [1.6\%]).

The median age for all patients was 64 years $\left(25^{\text {th }}-75^{\text {th }}\right.$ percentiles; $51-75$ years $)$ and $59.0 \%$ were male. Respiratory $(17.1 \%)$, urogenital (10.5\%), and digestive (10.2\%) tract infections accounted for more than one-third of consultations and $42.1 \%$ of patients had hospital-acquired infections.

Informal consultations were more likely to involve surgical or intensive care patients (Table 1) and patients with peripheral vascular disease (Table 2). Conversely, patients with urogenital tract infection or metastatic cancer were more likely to receive formal consultations. Other baseline characteristics did not differ between formal and informal consultations, resulting in a comparable Charlson comorbidity index score in the two groups.

Obtaining advice on antimicrobial therapy was a less common reason for requesting informal consultation than formal consultation. Conversely, informal consultations were more often requested for other reasons (Table 1). Residents were less likely than board-certified infectious disease specialists to provide informal consultations. Finally, informal consultations resulted less frequently in recommendations for antimicrobial treatment (178/184 [96.7\%] $v$ 
$443 / 443$ [100\%]; $P=0.001)$ and for diagnostic or monitoring tests $(117 / 184$ [63.6\%] $v$

322/443 [72.7\%]; $P=0.02$ ) than formal consultations.

Overall, the primary effectiveness outcome was assessed for 621 and 439 consultations that resulted in recommendations for antimicrobial treatment and diagnostic or monitoring tests, respectively (Figure). In univariable analysis, the compliance rates with recommendations for antimicrobial treatment $(86.5 \% v 88.9 \% ; P=0.40)$ and with recommendations for performing diagnostic or monitoring tests $(72.6 \% v 72.0 \% ; P=0.90)$ did not differ between the two groups (Table 3). No significant association was found between the type of consultation and compliance with the consultant's recommendations in adjusted analysis (Table 3).

Subsequent consultation, length of stay, and in-hospital mortality were assessed in all 627 patients, whereas early clinical improvement was ascertained in 506 patients who were symptomatic at baseline (Figure). In comparison to formal consultation, informal consultation was associated with a longer length of stay and comparable rates of subsequent consultation, early clinical improvement, and in-hospital mortality (Table 3). The difference in length of stay resulted from delays in requesting informal consultations (Table 1). No significant differences in patient clinical outcomes persisted between formal and informal recipients in propensity score-adjusted analysis (Table 3). 


\section{DISCUSSION}

The impact of informal consultations on quality of care and patient safety has raised questions for decades $[1,24]$. In this respect, this prospective cohort study provided a unique opportunity to examine the effectiveness and safety of specialist consultations. The findings of this study suggest that informal consultations result in levels of compliance with recommendations comparable to formal consultations without affecting clinically relevant outcomes for inpatients.

Low to moderate rates of compliance with the consultant's recommendations have been consistently reported for various internal medicine subspecialties, including infectious diseases [25]. Suboptimal interphysician communication during the formal consultation process may explain this observation [26-28]. In contrast, direct interaction between physicians is a core feature of informal consultations and may facilitate effective communication [1]. In our study, compliance with recommendations did not differ according to the type of consultation. This finding suggests that compliance is not affected by the lack of written recommendations resulting from informal consultation.

The major concern with informal consultation is that the consulting physician makes recommendations without directly examining the patient $[6,29]$. Indeed, anecdotal evidence exists that requesting physicians may convey incomplete or inaccurate information on patient clinical history, which in turn may result in inappropriate consultant recommendations [8]. Reassuringly, no significant differences were found in early clinical improvement and mortality rates depending on the type of consultation in our study. The apparently increased median length of stay for informal consultation recipients resulted from delays in requesting 
consultation. In a previous analysis [17], compliance with recommendations for antimicrobial therapy was associated with a higher rate of early clinical improvement and a shorter length of stay, suggesting that patient clinical outcomes are determined more by compliance with the consultant's recommendations than by the type of consultation.

It should be noted that a substantial percentage of patients died $(7.3 \%)$ or required unplanned subsequent infectious disease specialist consultations (34.8\%) during their hospital stay in both study groups. This finding may reflect the severity of illness as well as inappropriate consultant recommendations since no relationship was found between compliance with recommendations and in-hospital mortality or subsequent consultations [17].

The characteristics of formal and informal consultations differed to some extent in our study, suggesting that informal consultations were limited to less complex cases. There are several potential explanations for this observation. First, informal consultation is less appropriate when the reason for consultation requires consideration of several clinical variables at the same time. Second, informal consultation may require more experience than formal consultation, explaining why residents were less likely to provide informal consultations in our study. As advocated by guidelines [8], we recommend the consultant feel free to examine the patient if he or she believes the question is too complex to be resolved by informal consultation.

The limitations of this study should be acknowledged. First, because the original study was not designed for this purpose, the reasons for requesting or providing informal rather than formal consultations were not available. 
Second, our study was underpowered to detect small but clinically significant differences in safety outcomes between formal and informal consultation recipient because the sample size was determined by the number of patients enrolled in the original study. Therefore, we found a nonsignificant $3.5 \%$ absolute difference in mortality between the two study groups.

Third, the route of consultation was not assigned randomly in this observational study and the propensity score can compensate only for imbalances in the baseline characteristics measured $[22,23]$. Hence, we cannot exclude that our findings were confounded by differences in severity of illness or unmeasured comorbidities. However, our study investigated an important question that is unlikely to be addressed by a randomized controlled trial.

Fourth, we cannot exclude misclassification of early clinical improvement despite the use of standardized criteria. However, a previous analysis showed that early clinical improvement was paralleled by a shorter length of stay [17], a study outcome that was less likely to be affected by assessment bias. Fifth, our study was conducted at a single university-affiliated hospital in France and the results may not extend to other settings with different referral patterns.

Despite these limitations, this study provides observational evidence that informal consultations result in comparable levels of compliance with consultant recommendations without altering patient outcomes. These findings do not support concerns regarding the effectiveness and safety of informal consultations in infectious disease practice. However, further study is needed to refine the criteria for requesting or providing informal rather than formal consultations. 


\section{REFERENCES}

1. Golub RM. Curbside consultations and the viaduct effect. JAMA 1998;280(10):929930.

2. Forrest CB. A typology of specialists' clinical roles. Arch Intern Med 2009;169(11):1062-1068.

3. Gennai S, Francois P, Sellier E, Vittoz JP, Hincky-Vitrat V, Pavese P. Prospective study of telephone calls to a hotline for infectious disease consultation: analysis of 7,863 solicited consultations over a 1-year period. Eur J Clin Microbiol Infect Dis. 2010 Nov 11. [Epub ahead of print]

4. Kuo D, Gifford DR, Stein MD. Curbside consultation practices and attitudes among primary care physicians and medical subspecialists. JAMA 1998;280(10):905-909.

5. Pearson SD, Moreno R, Trnka Y. Informal consultations provided to general internists by the gastroenterology department of an HMO. J Gen Intern Med 1998;13(7):435-438.

6. Borowsky SJ. What do we really need to know about consultation and referral? J Gen Intern Med 1998;13(7):497-498.

7. Keating NL, Zaslavsky AM, Ayanian JZ. Physicians' experiences and beliefs regarding informal consultation. JAMA 1998;280(10):900-904.

8. Manian FA, Janssen DA. Curbside consultations. A closer look at a common practice. JAMA 1996;275(2):145-147.

9. Fox BC, Siegel ML, Weinstein RA. "Curbside" consultation and informal communication in medical practice: a medicolegal perspective. Clin Infect Dis 1996;23(3):616-622.

10. Duncan CJ, Gallacher K, Kennedy DH, Fox R, Seaton RA, MacConnachie AA. Infectious disease telephone consultations: Numerous, varied and an important educational resource. J Infect 2007;54(5):515-516. 
11. Leblebicioglu H, Akbulut A, Ulusoy S, et al. Informal consultations in infectious diseases and clinical microbiology practice. Clin Microbiol Infect 2003;9(7):724-726.

12. Schlesinger Y, Paltiel O, Yinnon AM. Analysis and impact of infectious disease consultations in a general hospital. J Hosp Infect 1998;40(1):39-46.

13. Yinnon AM. Whither infectious diseases consultations? Analysis of 14,005 consultations from a 5-year period. Clin Infect Dis 2001;33(10):1661-1667.

14. Fowler VG, Jr., Sanders LL, Sexton DJ, et al. Outcome of Staphylococcus aureus bacteremia according to compliance with recommendations of infectious diseases specialists: experience with 244 patients. Clin Infect Dis 1998;27(3):478-486.

15. Ballard WP, Gold JP, Charlson ME. Compliance with the recommendations of medical consultants. J Gen Intern Med 1986;1(4):220-224.

16. Sears CL, Charlson ME. The effectiveness of a consultation. Compliance with initial recommendations. Am J Med 1983;74(5):870-876.

17. Sellier E, Pavese P, Gennai S, Stahl JP, Labarere J, Francois P. Factors and outcomes associated with physicians' adherence to recommendations of infectious disease consultations for inpatients. J Antimicrob Chemother 2010;65(1):156-162.

18. von Elm E, Altman DG, Egger M, Pocock SJ, Gotzsche PC, Vandenbroucke JP. The Strengthening the Reporting of Observational Studies in Epidemiology (STROBE) statement: guidelines for reporting observational studies. Ann Intern Med 2007;147(8):573-577.

19. Charlson ME, Pompei P, Ales KL, MacKenzie CR. A new method of classifying prognostic comorbidity in longitudinal studies: development and validation. J Chronic Dis 1987;40(5):373-383.

20. Eron LJ, Passos S. Early discharge of infected patients through appropriate antibiotic use. Arch Intern Med 2001;161(1):61-65. 
21. Halm EA, Fine MJ, Marrie TJ, et al. Time to clinical stability in patients hospitalized with community-acquired pneumonia: implications for practice guidelines. JAMA 1998;279(18):1452-1457.

22. D'Agostino RB, Jr. Propensity score methods for bias reduction in the comparison of a treatment to a non-randomized control group. Stat Med 1998;17(19):2265-2281.

23. Joffe MM, Rosenbaum PR. Invited commentary: propensity scores. Am J Epidemiol 1999;150(4):327-333.

24. Myers JP. Curbside consultation in infectious diseases: a prospective study. $J$ Infect Dis 1984;150(6):797-802.

25. Lo E, Rezai K, Evans AT, et al. Why don't they listen? Adherence to recommendations of infectious disease consultations. Clin Infect Dis 2004;38(9):1212-1218. 26. Conley J, Jordan M, Ghali WA. Audit of the consultation process on general internal medicine services. Qual Saf Health Care 2009;18(1):59-62.

27. Salerno SM, Hurst FP, Halvorson S, Mercado DL. Principles of effective consultation: an update for the 21st-century consultant. Arch Intern Med 2007;167(3):271-275.

28. McPhee SJ, Lo B, Saika GY, Meltzer R. How good is communication between primary care physicians and subspecialty consultants? Arch Intern Med 1984;144(6):12651268.

29. Fox BC. Medical-legal aspects of curbside consultation. JAMA 1996;276(20):16351636. 
Figure. Patient enrollment.

Consultations resulting in no recommendations for antimicrobial treatment $(n=6)$ and diagnostic or monitoring tests $(n=188)$ were omitted from the analysis of compliance. Seventy patients who were asymptomatic at baseline, 49 patients who were discharged within $72 \mathrm{~h}$ of the index consultation, and two patients with missing values were omitted from the analysis of early clinical improvement. 
Table 1. Comparison of baseline characteristics between formal and informal infectious disease specialist consultations.

\begin{tabular}{|c|c|c|c|}
\hline \multirow[b]{2}{*}{ Characteristics } & \multicolumn{2}{|c|}{ ID specialist consultation } & \multirow[b]{2}{*}{$P$} \\
\hline & $\begin{array}{l}\text { Formal } \\
(N=443)\end{array}$ & $\begin{array}{l}\text { Informal } \\
(N=184)\end{array}$ & \\
\hline Male gender, $n(\%)$ & $259(58.5)$ & $111(60.3)$ & 0.67 \\
\hline Age, $y$, median $\left(25^{\text {th }}-75^{\text {th }}\right.$ percentiles $)$ & $64(51-76)$ & $63(51-74)$ & 0.64 \\
\hline Department, $n(\%)$ & & & $<0.001$ \\
\hline Medicine & $253(57.1)$ & $82(44.6)$ & \\
\hline Surgery & $185(41.8)$ & $91(49.5)$ & \\
\hline Intensive Care Unit & $5(1.1)$ & $11(6.0)$ & \\
\hline Time from admission, $\mathrm{d}$, median $\left(25^{\text {th }}-75^{\text {th }}\right.$ percentiles $)$ & $5(2-12)$ & $8(2-16)$ & 0.007 \\
\hline Weekend consultations, $n(\%)$ & $16(3.6)$ & $11(6.0)$ & 0.18 \\
\hline Resident versus board-certified ID specialist, $n(\%)$ & $338(76.3)$ & $79(42.9)$ & $<0.001$ \\
\hline \multicolumn{4}{|l|}{ Reason for requesting ID consultation, $n(\%)$} \\
\hline Antimicrobial therapy & $416(93.9)$ & $158(85.9)$ & 0.001 \\
\hline Diagnosis & $51(11.5)$ & $31(16.8)$ & 0.07 \\
\hline Other $^{\mathrm{a}}$ & $5(1.1)$ & $15(8.1)$ & $<0.001$ \\
\hline \multicolumn{4}{|l|}{ Diagnosis, $n(\%)$} \\
\hline Respiratory tract infection & $77(17.4)$ & $30(16.3)$ & 0.74 \\
\hline Urogenital tract infection & $55(12.4)$ & $11(6.0)$ & 0.02 \\
\hline Digestive tract infection & $43(9.7)$ & $21(11.4)$ & 0.52 \\
\hline Surgical site infection & $47(10.6)$ & $13(7.1)$ & 0.17 \\
\hline Soft tissue infection & $32(7.2)$ & $14(7.6)$ & 0.87 \\
\hline Bone and joint infection & $29(6.5)$ & $14(7.6)$ & 0.63 \\
\hline Bacteremia/sepsis & $34(7.7)$ & $8(4.3)$ & 0.13 \\
\hline Central nervous system infection & $22(5.0)$ & $12(6.5)$ & 0.43 \\
\hline Other & $64(14.4)$ & $31(16.8)$ & 0.44 \\
\hline Hospital-acquired infection, $n(\%)^{\mathrm{b}}$ & $183(41.3)$ & $81(44.3)$ & 0.50 \\
\hline Ongoing antimicrobial treatment, $n(\%)$ & $301(67.9)$ & $120(65.2)$ & 0.51 \\
\hline Contact isolation for infection control, $n(\%)$ & $43(9.7)$ & $26(14.1)$ & 0.11 \\
\hline
\end{tabular}


Abbreviations: ID, infectious disease

${ }^{a}$ Other reason for requesting ID consultation included antimicrobial prophylaxis $(n=9)$ and patient follow-up after discharge $(n=11)$.

${ }^{\mathrm{b}}$ Information on hospital-acquired infection status was missing for one patient 
Table 2. Comparison of patient comorbid diseases and Charlson index score between formal and informal infectious disease specialist consultations.

\begin{tabular}{|c|c|c|c|}
\hline & \multicolumn{2}{|c|}{ ID specialist consultation } & \multirow[b]{2}{*}{$P$} \\
\hline & $\begin{array}{l}\text { Formal } \\
(N=443)\end{array}$ & $\begin{array}{l}\text { Informal } \\
(N=184)\end{array}$ & \\
\hline \multicolumn{4}{|l|}{ Comorbid diseases, $n(\%)$} \\
\hline Congestive heart failure & $58(13.1)$ & $35(19.0)$ & 0.06 \\
\hline Nonmetastatic cancer & $64(14.4)$ & $26(14.1)$ & 0.92 \\
\hline Metastatic cancer & $35(7.9)$ & $5(2.7)$ & 0.02 \\
\hline Peripheral vascular disease & $50(11.3)$ & $35(19.0)$ & 0.01 \\
\hline Renal failure & $49(11.1)$ & $26(14.1)$ & 0.28 \\
\hline Diabetes, uncomplicated & $48(10.8)$ & $15(8.1)$ & 0.31 \\
\hline Diabetes, complicated & $32(7.2)$ & $16(8.7)$ & 0.53 \\
\hline Chronic pulmonary disease & $41(9.3)$ & $16(8.7)$ & 0.82 \\
\hline Cerebrovascular disease & $39(8.8)$ & $15(8.1)$ & 0.79 \\
\hline Liver disease & $33(7.4)$ & $13(7.1)$ & 0.87 \\
\hline Psychiatric disorder & $29(6.5)$ & $14(7.6)$ & 0.63 \\
\hline Myocardial infarction & $33(7.4)$ & $19(10.3)$ & 0.23 \\
\hline Alcohol abuse & $32(7.2)$ & $7(3.8)$ & 0.11 \\
\hline Fluid and electrolyte disorders & $24(5.4)$ & $7(3.8)$ & 0.40 \\
\hline Hypothyroidism & $20(4.5)$ & $8(4.3)$ & 0.93 \\
\hline Paralysis & $18(4.1)$ & $7(3.8)$ & 0.88 \\
\hline Obesity & $12(2.7)$ & $10(5.4)$ & 0.09 \\
\hline Lymphoma & $11(2.5)$ & $10(5.4)$ & 0.06 \\
\hline Peptic ulcere disease & $16(3.6)$ & $3(1.6)$ & 0.19 \\
\hline Coagulopathy & $13(2.9)$ & $5(2.7)$ & 0.88 \\
\hline Rheumatoid arthritis/CVD & $13(2.9)$ & $4(2.2)$ & 0.79 \\
\hline Dementia & $10(2.3)$ & $3(1.7)$ & 0.76 \\
\hline AIDS & $4(0.9)$ & $3(1.6)$ & 0.42 \\
\hline
\end{tabular}

(Continued on next page) 
Table 2. (Continued)

\begin{tabular}{|c|c|c|c|}
\hline & \multicolumn{2}{|c|}{ ID specialist consultation } & \multirow[b]{2}{*}{$P$} \\
\hline & $\begin{array}{l}\text { Formal } \\
(N=443)\end{array}$ & $\begin{array}{l}\text { Informal } \\
(N=184)\end{array}$ & \\
\hline Drug abuse & $2(0.4)$ & $4(2.2)$ & 0.06 \\
\hline Leukemia & $2(0.4)$ & $2(1.1)$ & 0.58 \\
\hline Charlson index score, $n(\%)^{\mathrm{a}}$ & & & 0.52 \\
\hline 0 & $168(37.9)$ & $62(33.7)$ & \\
\hline $1-2$ & $132(29.8)$ & $57(31.0)$ & \\
\hline $3-4$ & $70(15.8)$ & $37(20.1)$ & \\
\hline$\geq 5$ & $73(16.5)$ & $28(15.2)$ & \\
\hline
\end{tabular}

Abbreviations: CVD, collagen vascular diseases; AIDS, acquired immune deficiency syndrome

${ }^{a}$ Charlson index score was computed based on the standardized definitions and weights for the 19 comorbid diseases that comprised this score [16]. 
Table 3. Comparison of compliance with recommendations and patient clinical outcomes between formal and informal infectious disease specialist consultations

ID specialist consultation

Formal Informal

Primary effectiveness outcomes, $n / N(\%)^{\mathrm{b}}$

Compliance with recommendations for

$394 / 443(88.9) \quad 154 / 178(86.5)$

$0.80(0.47-1.35)$

treatment

Compliance with recommendations for

$232 / 322(72.0)$

$85 / 117(72.6)$

$1.03(0.64-1.66)$

performing diagnostic or monitoring tests

Patient clinical outcomes, $n / N(\%)$

Subsequent ID specialist consultation ${ }^{\mathrm{c}}$

$161 / 443(36.3) \quad 63 / 184(34.2)$

$0.91(0.64-1.31)$

Early clinical improvement ${ }^{\mathrm{d}}$

$218 / 372(58.6)$

$78 / 134(58.2)$

$0.99(0.66-1.47)$

In-hospital mortality

$37 / 443(8.4)$

9/184 (4.9)

$0.56(0.27-1.20)$

Length of stay, $d$, median $\left(25^{\text {th }}-75^{\text {th }}\right.$

$20(10-32)$

$23(11-35)$

$0.80(0.67-0.95)$

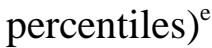

Abbreviation: ID, infectious disease

${ }^{\text {a }}$ Odds ratios were adjusted for quintile of propensity score. Our propensity score included all baseline characteristics and comorbid diseases (with the exception of the Charlson comorbidity index score) listed in Tables 1 and 2.

${ }^{\mathrm{b}} \mathrm{Six}$ consultations resulting in no recommendation for antimicrobial treatment and 188 consultations resulting in no recommendation for performing diagnostic or monitoring tests were omitted from the analysis of compliance.

${ }^{\mathrm{c}}$ Subsequent consultations were unplanned for 218 patients and planned for six patients. 
${ }^{\mathrm{d}}$ Seventy patients who were asymptomatic at baseline, 49 patients who were discharged within $72 \mathrm{~h}$ of index consultation, and two patients with missing values were omitted from the analysis of early clinical improvement.

${ }^{\mathrm{e}}$ A discrete time logistic hazard model was used to examine the association between the length of stay and the type of consultation. The length of stay was censored at the time of death for patients who died in the hospital. A lower odds of discharge corresponded to a longer length of stay. 